\title{
Assessment of Fetal and Infant health status based on Non-Stress Test
}

\author{
Research Article
}

\section{Monireh Rezaee Moradali ${ }^{1}$, Moradali Zareipour ${ }^{2}$, Zhila Mohammad Rezaei $^{*}$}

\author{
1. PhD Student of Reproductive Health, Student Research Committee, School of Nursing and Midwifery, \\ Shahid Beheshti University of Medical Sciences, Tehran, Iran \\ 2. $\mathrm{PhD}$ of Health Education and Health Promotion, Health System Research Unit, Urmia Health Center, \\ Urmia University of Medical Sciences, Urmia, Iran \\ 3. MSc in midwifery, Department of Midwifery, Urmia Branch, Islamic Azad University, Urmia, Iran
}

\begin{abstract}
Introduction: Non stress test (NST) is commonly considered as a screening test to assess the health of the fetus especially in high risk pregnancies in most studies. The purpose of this study was to Assessment of fetal and Infant health status based on Non-Stress Test. Methods: This study was a case-control design carried out on 984 pregnant in Urmia selected by census sampling during 2014-2015; 500 pregnant had experienced NST and considered as case group and 484 pregnant had not experienced NST and considered as control group. Data were collected using records of NST among mothers and checklist including variables related to maternal characteristics such as fetal characteristics. The data were analyzed through SPSS version 22 software. Results: In pregnant women who experienced NST, the mean age, Apgar min 1 and 5 was significantly different and NST group had better situation than other group $(\mathrm{p}=0.001)$. Fetal distress was more in the presence of meconium $(\mathrm{p}=0.0001)$. In binary logistic regression analysis, meconium and NST were presented as a risk factor in the model, that is, by shifting one level of the meconium or NST status (yes/no) $\left(\mathrm{OR}_{\text {Meconium }}=14.262, \mathrm{p}=0.0001\right.$; OR NST $=2.874, \mathrm{p}=$ 0.001). Conclusion: Using non stress test to observe the abnormal results of the fetus especially meconium-stained amniotic fluid is crucial and vital because can prevent the fetal distress, infection of pregnant women and fetus and even the mortality of the fetus.
\end{abstract}

Keywords: Non stress test, Fetal, Infant Health Status.

\section{Introduction}

Perinatal events play a major role in neonatal mortality (1) Thirty percent of perinatal deaths are due to placenta complication, such as infection, hydrops, meconium aspiration, and maternal complications including diabetes mellitus and hypertension (2). Meconium excretion is one of the symptoms of fetal distress and meconium-stained amniotic fluid is happen in $7 \%$ to $22 \%$ of pregnant women (3).

An infant exposed to meconium is at increased risk for meconium aspiration, pulmonary disease and pneumothorax, and appropriate opportunity for the growth of bacteria (4). Women with meconium-stained amniotic fluid suffer from fever and infection after childbirth (5). It is estimated that more than nine million neonate or fetus die in the early weeks of their birth or before birth each year, and almost all of these deaths occur in developing countries (6). The term "fetal distress" indicates the condition of the fetus that has changed in such a way that early death or permanent injuries may occur in a short time. Naturally, the determination of these conditions is drastically required for the survival of the fetus, and timely action is necessary (7).

*Corresponding Author:

\section{Zhila Mohammad Rezaei}

MSc in midwifery,

Department of Midwifery, Urmia Branch,

Islamic Azad University, Urmia, Iran

Email: j.mrezayi@yahoo.com
Over the past two decades, with a thorough knowledge of the human embryo, along with relevant technological advances, a new phenomenon has emerged in midwifery that predicts the health of the fetus. For this reason, several types of embryonic health examination methods have been developed with specific characteristics and predictive values $(8,9)$. Some of these methods include fetal movement control, nonstress test, contractility test, and biophysical profiles (10).

In fact, fetal health tests have become an important part of midwifery care $(8,9)$. Nowadays, NST is the most common and the first recommended test to check the health of the fetus in most studies $(10,11)$ that are used in high risk pregnancies. Based on the results of this test, it is possible to decide on the continuation or termination of pregnancy and to provide appropriate ways to reduce fetal mortality, maternal mortality and economic and psychological damage (10). NST is a standard method for pre-natal evaluation due to the simplicity and lack of contraindication (12). The purpose of this study was to Assessment of fetal and Infant health status based on Non-Stress Test.

\section{Materials and Methods}

This study was a case-control design carried out on 984 pregnant in Urmia selected by census sampling during 2014-2015; 500 pregnant had experienced NST and considered as case group and 484 pregnant had not experienced NST and considered as control group.

Data collection method, Maternal case study The 
data collection tool was a researcher-made checklist based on previous studies that verified and verified the validity and reliability. In this research, in order to achieve the research goals, the checklist, based on the information in the books, articles and publications.

Data were collected using records of NST among mothers. The checklist included 16 variables checklist including variables related to maternal characteristics such as fetal characteristics.

Inclusion criteria were mothers of fetus without genetic abnormalities and available NST in the week after 32 gestational age. Exclusion criteria were multiple pregnancies, a history of maternal preeclampsia, smoking, placental abruption, diabetes, ectopic pregnancy, collagen vascular diseases, molar pregnancy and hydrops fetalis.

This project was approved by ethic committee of Islamic Azad University, Urmia branch (IR.IAU.URMIA.REC.1396.08). and the goals of the study were explained to all parents and all of them accepted to participate and were assured consider the confidentiality of their individual information as well as the voluntary nature of participating in the study.

The collected data was analyzed using SPSS version.22 software. Descriptive analysis was used to determine the distribution of absolute frequency and percentages, mean and standard deviation of variables, and inferential analysis (Chi-Square, linear regression, logistic regression tests) was used to determine the relationships between the independent variables and dependent variable.

\section{Results}

In the present study, the amniotic fluid exposure to meconium was more in non-reactive NST compare to reactive NST (14.3\% vs. $2.7 \%$ ) showing that meconium can affect the normal action of sympathetic and parasympathetic nervous system, and the statistical test showed a significant relationship between non-reactive NST and meconium exposure. Also, in this study, the rate of fetal distress in centers with NST was approximately twice that of centers without it and when this test was non-reactive, the amount of fetal distress was more $(80.8 \%$ vs. $19.2 \%)$; statistical test also showed a significant relationship between non-reactive NST and fetal distress (Table 1 and 2). Fetal distress was more in the presence of meconium $(p=0.0001)$. In pregnant women who experienced NST, the mean age, Apgar min 1 and 5 was significantly different and NST group had better situation than other group $(\mathrm{p}=0.001)$ (Table 3).

In binary logistic regression analysis, meconium and NST were presented as a risk factor in the model, that is, by shifting one level of the meconium or NST status (yes/no), provided that the other variables remain constant in the model $(\mathrm{OR}$ Meconium $=14.262, \mathrm{p}=0.0001$; $\left.\mathrm{OR}_{\mathrm{NST}}=2.874, \mathrm{p}=0.001\right)($ Table 4$)$.

Table 1: Frequency of fetal distress and neonate status and the result of NST in delivery centers

\begin{tabular}{|c|c|c|c|c|c|c|}
\hline \multirow{2}{*}{\multicolumn{2}{|c|}{ Variable }} & \multicolumn{2}{|l|}{ Reactive } & \multicolumn{2}{|c|}{ Non-reactive } & \multirow[t]{2}{*}{ P value } \\
\hline & & Frequency & Percent & Frequency & Percent & \\
\hline \multirow[t]{2}{*}{ Meconium } & Yes & 9 & 2.7 & 19 & 14.3 & \multirow[t]{2}{*}{0.001} \\
\hline & No & 328 & $\overline{97.3}$ & 114 & 85.7 & \\
\hline \multirow[t]{2}{*}{ Fetal distress } & Yes & 14 & 4.1 & 59 & 43.1 & \multirow[t]{2}{*}{0.001} \\
\hline & No & 331 & $\overline{95.9}$ & 78 & 56.9 & \\
\hline
\end{tabular}

Table 2: Frequency of fetal distress and meconium status centers

\begin{tabular}{|l|l|c|c|c|c|l|}
\hline \multicolumn{2}{|l|}{ Variable } & \multicolumn{2}{|c|}{ Meconium } & \multirow{2}{*}{ Total } & \multirow{2}{*}{ P value } & \multirow{2}{*}{$\mathbf{X}^{2}$} \\
\cline { 3 - 5 } \multicolumn{2}{|c|}{ Fetal distress } & Yes & No & & & \\
\cline { 2 - 5 } & No & 817 & 34 & 109 & $<0.0001$ & 102.790 \\
\cline { 2 - 5 } & Total & 76 & 33 & 851 & & \\
\hline
\end{tabular}

Table 3: Comparison of the mean age, weight, Apgar min 1 and 5 in the both group of NST and without NST

\begin{tabular}{|c|c|c|c|c|c|}
\hline \multicolumn{2}{|c|}{ Variable } & Mean & Std. Deviation & $\mathbf{t}$ & P value \\
\hline \multirow{2}{*}{ Age } & NST & 27.56 & 6.53 & \multirow[t]{2}{*}{-2.945} & \multirow[t]{2}{*}{0.003} \\
\hline & Without NST & 28.79 & 6.49 & & \\
\hline \multirow{2}{*}{ Weight } & NST & 3189.65 & 467.66 & \multirow{2}{*}{1.175} & \multirow[t]{2}{*}{0.080} \\
\hline & Without NST & 3139.25 & 426.15 & & \\
\hline \multirow{2}{*}{ Apgar $1 \mathrm{~min}$} & NST & 8.65 & 0.61 & \multirow[t]{2}{*}{3.634} & \multirow[t]{2}{*}{0.001} \\
\hline & \begin{tabular}{|l} 
Without NST \\
\end{tabular} & 8.46 & 1.00 & & \\
\hline \multirow{2}{*}{ Apgar 5 min } & NST & 9.65 & 0.61 & \multirow[t]{2}{*}{4.110} & \multirow[t]{2}{*}{0.001} \\
\hline & \begin{tabular}{|l} 
Without NST \\
\end{tabular} & 9.44 & 0.94 & & \\
\hline
\end{tabular}


Table 4: Predictive variables for development of distress (as dichotomous variable: with/without distress) using binary logistic regression

\begin{tabular}{|l|c|c|c|c|c|}
\hline Variable & Odds ratio & Std. error & P value & Lower limit & Upper limit \\
\hline Meconium & 14.262 & 0.301 & $\mathbf{0 . 0 0 1}$ & 7.781 & 25.404 \\
\hline NST & 2.874 & 0.246 & $\mathbf{0 . 0 0 1}$ & 1.803 & 4.713 \\
\hline Age & 0.984 & 0.017 & 0.317 & 0.951 & 1.015 \\
\hline Weight group (level 2) & 2.599 & 1.042 & 0.359 & 1.166 & 7.736 \\
\hline Weight & 1.001 & 0.000 & 0.038 & 1.000 & 1.001 \\
\hline Gender & 0.906 & 0.229 & 0.665 & 0.591 & 1.442 \\
\hline
\end{tabular}

In this study, the main reason for the NST test was Premature Rupture of Membranes (PROM) (41.7\%), followed by post-date, meconium excretion, and decreased fetal movements and post-term. The results of the study by Modarres et al. on NST indication showed that some units had more than one indication. The most indications were decreased fetal movement $(25 \%)$, a history of fetal death $(22.5 \%)$, fetus examination according to hospital protocol $(17.5 \%)$, developing gestational diabetes mellitus and a history of infertility $(15 \%)$, pre-pregnancy diabetes and pre-eclampsia $(7.5 \%)$, heart disease $(5 \%)$ and mother's high age $(5 \%)$, polyhydramnios, prolonged pregnancy and excessive maternal weight and cesarean section due to heart rate decrease in the first child (2.5\%) (13).

In the recent study, the meconium-stained amniotic fluid had more non-reactive NST than -reactive NST (14.3\% vs. $2.7 \%)$, and the statistical test showed a significant relationship between non-reactive NST and meconium. In this study, the rate of fetal distress in centers with NST was approximately twice that of centers without it, and when this test was non-reactive, the amount of fetal distress was higher $(80.8 \%$ vs. 19.2 $\%)$. A study by Naderi et al. showed that $62 \%$ of nonreactive NST had fetal distress (14). Meconium excretion is one of the symptoms of fetal distress showing hazardous situation of fetus, in which requires urgency appropriate intervention. Actually meconiumstained amniotic fluid increases the chance of chorioamnionitis with high prevalence (15). A study by Rahimi et al., carried out on 573 pregnant on the relationship between meconium amniotic fluid and fever and postpartum infection and the results showed that 82 $(14 \%)$ were febrile and the fever was longer than 24 hours in in the control group. Wound infection was $7.1 \%$ in the meconium group versus $0.68 \%$ in the control group. Therefore the presence of meconium is an independent risk factor for postpartum endometritis (16).

In this study, there was a statistically significant relationship between NST with the majority of the components of fetal-neonatal-maternal outcomes such as fetal death, meconium staining, fetal distress, low birth weight, Apgar score, neonatal death and abnormal movement test in labor centers. By observing the abnormal results of this test, midwives in these centers can quickly decide on appropriate referrals of mothers to hospitalized centers and prevent mothers from developing adverse of fetus and neonatal symptoms . Awareness of the results of this research can be helpful in providing patients with services to the staff of labor facilities, so that in dealing with pregnant mothers indicating a non-reactive NST, they can save time and cost of patients by doing the test as soon as possible. It can reduce the anxiety of pregnant mothers without performing unnecessary interventions before the test; they prevent the risk of a possible fetal distress.

However, with the referral of mothers to hospitals and non-reactive NST test outcomes, the rate of cesarean section increases so that they can quickly save the life of the neonates a t risk danger, but it seems that the incidence of cesarean section can be used by higher sensitivity tests and maternal monitoring in the hospital's facilities .Regarding the importance of the correct interpretation of the NST test, the class of NST test interpretation should be held in the health centers, as well as in the universities in the maternity, midwifery and obstetrics courses for midwifery students. As well as instructors will provide students with the necessary training in this regard.

\section{Limitations of the study}

One of the limitation points of the study is the design of the study (retrospective) and referral to mothers' records. Some cases were incomplete, which reduced the number of samples.

\section{Conclusion}

Using non stress test to observe the abnormal results of the fetus especially meconium-stained amniotic fluid is crucial and vital because can prevent the fetal distress, infection of pregnant women and fetus and even the mortality of the fetus. This study found the strong relationship between meconium-stained amniotic fluid and distress.

\section{Acknowledgements}

This research project (\#24278) was funded by Urmia Branch, Islamic Azad University. The authors would like to thank the Vice-chancellor for Research \& Technology of Ardabil University of Medical Sciences and all contributors of this project.

\section{Reference}

1. Cuningham FG, Leveno KJ, Bloom SL, Williams JW. Intrapartum assessment. In: Williams Obstetrics.23nd ed. New York: Mc GrawHill;2010.

2. Gabbe SG, Niebyl JR, Simpson JL, Galan H, Goetzl L, Jauniaux ERM. Obstetrics: Normal andproblem pregnancies. 5th ed. Philadelphia: Churchill Livingstone; 2007. 
3. Nathan L, Leveno KJ, Carmody TJ 3rd, Kelly MA, Sherman ML. Meconium: a 1990s perspective on an old obstetric hazard. Obstet Gynecol 1994; 83: 329-32.

4. Matthews TG, Warshaw JB. Relevance of the gestational age distribution of meconium passage in utero. Pediatrics 1979; 64: 30- 1.

5. Clurk P, Doff P. Inhibition of neutroplil Oxidative burst and phagosytosis by meconium. Am J Obstet Gynecol 1994; 143: 1301- 5.

6. World Health Organization: Causes of stillbirths and early neonatal deaths: data from7993 pregnancies in six Developing Countries, 2006; 84(9): 699-705.

7. Spong GY. Fetal monitoring. In: Scott JR, Gibbs RS, Karlan BY, Haney AF, editors. Danforth's Obstetrics Gynecology. 9th ed. Philadelphia: Lippincott Williams \& Wilkins; 2003. p. 159-92.

8. Baskett TF. Gestational age and fetal biophysical assessment. Am J Obstet Gynecol. 1988;158(2): 332-4.

9. Vintzileos AM, Gaffney SE, Salinger LM, Campbell WA,Nochimson DJ. The relationship between fetal biophysical profileand cord $\mathrm{pH}$ in patients undergoing cesarean section before theonset of labor. Obstet Gynecol 1987; 70: 196201.

10. Cunningham FG, Leveno KJ, Bloom SL, et al, editor. Williams Obstetrics. $24^{\text {th }}$ Ed. New York: MC Graw Hill; 2014.
11. Roger K, Freeman Thomas J, Garite MI. Nageotte "Fetal Heart Monitoring" 2 nd ed, 1991; pp: 115792.

12. Benedict A, O'riordan M, Kirchner H, lester Shah D , Hack M Perinatal Correlates and neonatal morbidity associated with intrauterine growth factor among neonates or of term gestation. Am J Obstet Gynecol. 2001; 185 (3): 652- 59.

13. Modarres M, Mir Mohammad Ali M, Haghani H, Arami R, Rahnama P. The conformity of BPP and vibroacoustic stimulation results in fetal nonreactive non stress test. Tehran Univ Med J. 2006; 64 (9) :46-54.

14. Naderi T, Nikian Y, Amin Zadeh F. Sensitivity and specificity of NST in cases with decrease of fetal movement and amniotic fluid volume. JBUMS. 2002; 4 (1) :7-10.

15. Romero R, Hanaoka S, Mazor M, Athanassiadis AP, Callahan R, Hsu YC, et al. Meconium-stained amniotic fluid: a risk factor for microbial invasion of the amniotic cavity. Am J Obstet Gynecol 1991; 164: 859-62.

16. Rahimi Sharbaf F, Davari Tanha F, Niromansh Sh, Salehi N, Valadan $\mathrm{M}$, Niromand $\mathrm{N}$ et al . Meconium-stained amniotic fluid as an independent risk factor for fever and postpartum infection in term pregnancy. Tehran Univ Med J. 2008; 66 (3) :203-207 